\title{
Interfaces
}

INTERFACES Image Texte Language

$45 \mid 2021$

Jeux de formats (1)

\section{Les Micro-Salons de la Galerie Iris Clert entre 1957 et 1980}

Servin Bergeret

\section{(2) OpenEdition}

1 Journals

\section{Édition électronique}

URL : https://journals.openedition.org/interfaces/2630

DOI : 10.4000/interfaces.2630

ISSN : 2647-6754

Éditeur :

Université de Bourgogne, Université de Paris, College of the Holy Cross

\section{Édition imprimée}

Date de publication : 12 juillet 2021

ISSN : 1164-6225

\section{Référence électronique}

Servin Bergeret, "Les Micro-Salons de la Galerie Iris Clert entre 1957 et 1980 », Interfaces [En ligne], 45 | 2021, mis en ligne le 12 juillet 2021, consulté le 16 septembre 2021. URL : http://

journals.openedition.org/interfaces/2630; DOI : https://doi.org/10.4000/interfaces.2630

Ce document a été généré automatiquement le 16 septembre 2021.

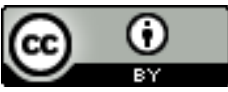

Les contenus de la revue Interfaces sont mis à disposition selon les termes de la Licence Creative Commons Attribution 4.0 International. 


\title{
Les Micro-Salons de la Galerie Iris Clert entre 1957 et 1980
}

\author{
Servin Bergeret
}

\section{À Louise Barbu}

1 Contrairement à l'idée communément admise par l'histoire de l'art, l'exposition des Propositions monochromes d'Yves Klein en mai $1957^{1}$ n'est pas la première manifestation artistique qui attira l'attention sur la galerie d'art contemporain dirigée par Iris Clert, 3 rue des Beaux-arts à Paris. En effet, c'est un mois plus tôt, en avril 1957, que la jeune galeriste accomplit son premier « coup » remarquable sur la scène du monde de l'art contemporain parisien en inaugurant son Micro-Salon d'Avril 1957. L'ambition de cette exposition originale repose sur la volonté d'Iris Clert d'accrocher dans un minimum d'espace un maximum d'œuvres. Pour ce faire, la galeriste passe commande auprès d'artistes d'œuvres de petits formats, appelés dans le jargon du métier : «format un figure » - pas plus de $22 \mathrm{~cm} \times 16 \mathrm{~cm}$, selon l'unité de mesure des toiles du commerce format que le collectionneur Maître Rey baptise à la suite d'un jeu de mots : «Les Huns » (Chupin-Morellet 18). Iris Clert parle de «tableau portable» (FIAC 1975 109) ou encore de "zéro figure " (Clert 1975, cassette $n^{\circ} 2$ ), en référence à l'appellation officiellement employée. Ces œuvres de petits formats permettent à la galeriste de relever le défi d'exposer simultanément près de deux cent cinquante œuvres dans moins de $15 \mathrm{~m}^{2}$.

2 Ce Micro-Salon d'Avril rencontre une réception critique immédiatement élogieuse et fait de la petite galerie d'Iris Clert l'un des nouveaux lieux d'exposition d'art contemporain alternatif parisien ; artistes, critiques d'art et autres collectionneurs lui resteront désormais fidèles ${ }^{2}$. Par ailleurs, ce format d'exposition, Micro-Salon, devient une marque de fabrique de la galerie qui en programme une douzaine entre 1957 et 1980.

Il convient d'explorer la valeur historique de ces "gestes de mise en exposition" (Davallon 11) ${ }^{3}$, dont la liberté repose exclusivement sur le petit format des œuvres exposées. Ainsi, il s'agit d'étudier le parti pris des discours qui émanent de certains de ces Micro-Salons ${ }^{4}$, dans le sens où ils témoignent de la place qu'Iris Clert a occupée dans 
l'espace de production artistique, à travers l'émergence d'une pensée sur l'art et la société, tout en mettant en évidence les choix de commissaire d'exposition faits par cette galeriste.

\section{Au salon des refusés}

4 L'origine de l'invention du Micro-Salon d'Avril 1957 est indissociable de l'importance du rôle que les Salons occupent dans le monde de l'art contemporain d'après-guerre. En effet, ces derniers vont contribuer à donner une visibilité aux avant-gardes d'une époque dominée par la Nouvelle École de Paris. Cette dernière, dans la diversité de ses langages formels et idéologiques, réveille de nombreux débats esthétiques entre les tenants de la figuration et ceux de l'abstraction. Des débats subsistent au sein même de l'abstraction, partagée en deux entités distinctes : une abstraction lyrique et une abstraction construite. Chacune va rencontrer une large réception critique, avoir ses émules, ses ardents défenseurs, mais aussi ses détracteurs. Les instances de représentation artistique participent à ce mouvement d'émulation créatrice, dont la plus active demeure le système des salons, qui s'évertue à réaffirmer sa place d'acteur collectif servant les partis pris esthétiques ${ }^{5}$. Ainsi, le Paris artistique connaît la naissance de nouveaux salons, parmi lesquels deux prédominent: le Salon des Réalités Nouvelles et le Salon de Mai. Initialement créé en 1939, le Salon des Réalités Nouvelles ne propose sa première édition au Palais des Beaux-Arts de la ville de Paris qu'en 1946. Imaginé par l'amateur d'art Fredo Sidès, et animé à ses débuts par Auguste Herbin et Félix Del Marle, ce salon s'impose en lien avec Abstraction-Création ${ }^{6}$ et consacre sa programmation uniquement à l'art abstrait concret, construit et non figuratif (D’Orgeval). Puis Le Salon de Mai ouvre ses portes dans les locaux de la nouvelle galerie Pierre Maurs le 29 mai 1945. Ce salon répond aux espérances de son fondateur, Gaston Diehl, qui compte réunir auprès d'artistes confirmés de jeunes talents représentatifs des diverses tendances existantes; il s'agit de propager « une même volonté de miser sur l'avenir de l'art » (Roig Sotomayor 38). Au fil des ans, ces deux salons font autorité au sein des débats artistiques, attisent les convoitises, puis, victimes de leur réussite, sont contraints de choisir de plus en plus sévèrement les œuvres, de sorte qu'ils apparaissent à la fin des années cinquante comme des instances officielles. L'histoire des salons reprend alors ses droits, avec l'émergence de dissidents, dont Les peintres témoins de leur temps en 1951 et, cinq ans plus tard, Comparaisons.

C'est dans ce contexte qu'Iris Clert propose, en avril 1957, une exposition originale pour devancer le Salon de Mai, qu'elle tient pour le plus influant et prisé de l'époque (Clert 2003, 140). Déplorant l'absence systématique, dans cet accrochage, des jeunes artistes en qui elle croit, elle décide de préparer une contre-manifestation audacieuse, qu'elle intitule avec éloquence : Le Micro-Salon d'Avril. Pour créer l'événement, la galeriste décide d'exposer dans sa petite galerie un maximum d'œuvres, mêlant les noms d'artistes reconnus à ceux de la jeune génération. Cherchant conseil auprès de ses proches pour relever ce défi, elle rapporte qu'un artiste lui aurait suggéré sur le ton de la plaisanterie : «Tu devrais nous demander de te faire des tableaux format carte postale » (140). La boutade prise au sérieux, elle explique s'être décidée à recueillir un ensemble, le plus important possible, d'œuvres de petits formats - «format un figure ": pas plus de $22 \mathrm{~cm} \times 16 \mathrm{~cm}$. Hormis ce témoignage, il semblerait que la conception de ce Micro-Salon d'Avril trouve sa source d'inspiration dans deux 
expositions successivement organisées en 1955 et 1956 à la Galerie La Roue de Guy Resse, qui, sous l'égide du critique d'art Michel Ragon, réunissaient un ensemble d'œuvres de petits formats sous le titre : Éloge du petit format ${ }^{7}$.

6 Le Micro-Salon d'Avril surprend pour avoir accueilli dans moins de $15 \mathrm{~m}^{2}$ et sur trois pans de murs près de deux cent cinquante œuvres (majoritairement des peintures et quelques sculptures) réalisées par cent quinze artistes - des hommes et quelques femmes - d'expressions et de générations différentes ${ }^{8}$ (Figure 1).

Figure 1. Carton d'invitation à l'exposition collective Micro-Salon d'Avril. Paris, Galerie Iris Clert, 3 rue des Beaux-Arts (12 avril - 8 mai 1957).

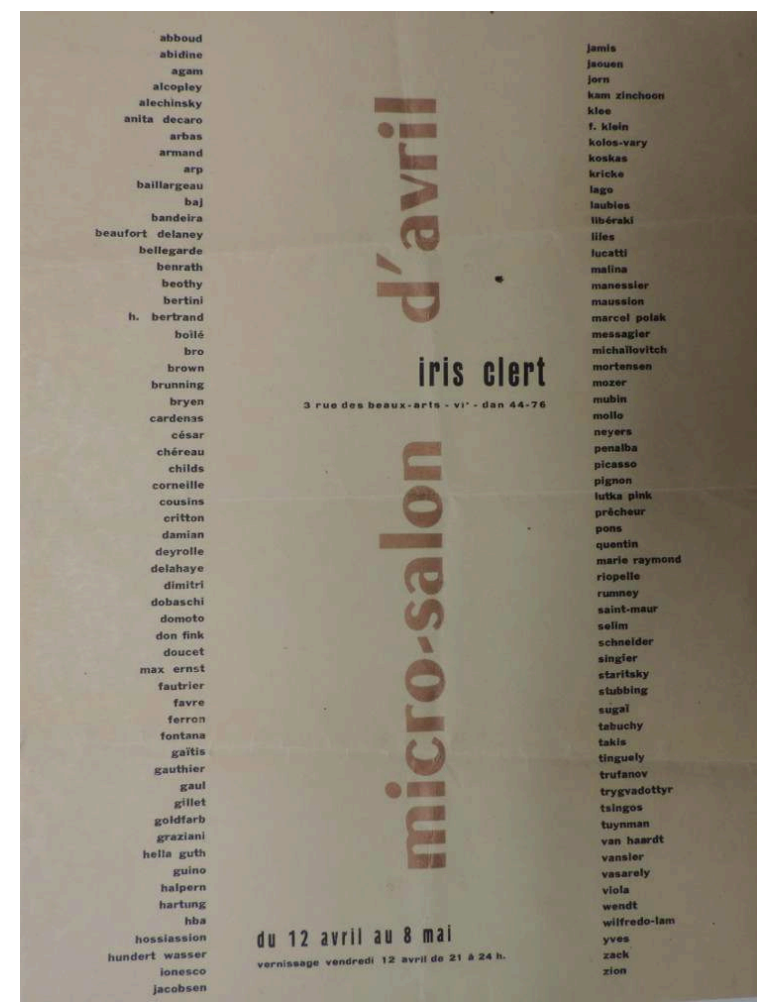

(C) Bibliothèque Kandinsky, MNAM/CCI, Centre Pompidou, Fonds Iris Clert.

7 L'éclectisme des œuvres mises en relation est comme tempéré par la composition de l'accrochage : la présence d'un monochrome d'Yves Klein au centre de chaque panneau vient donner un équilibre à l'ensemble (Clert 2003, 145). Cet agencement s'apparente à un pied-de-nez adressé indirectement au Salon des Réalités Nouvelles, qui, deux ans auparavant, avait refusé le monochrome orange de cet artiste, Expression de l'univers de la couleur mine orange ${ }^{9}$.

8 À bien le considérer, ce Micro-Salon d'Avril s'inscrit dans la tradition des Salons dissidents. Iris Clert reste fidèle à ce mode de fonctionnement et œuvre en marge et en dissidence ; le salon devient même l'un des partis pris majeurs de sa galerie et le plus représentatif de sa position vis-à-vis des espaces de représentation institutionnels et officiels de la vie artistique contemporaine de son temps. Ceci étant, en 1962, elle inventera la biennale OFF de Venise (Agostinelli 93). Le vernissage de sa Piccola Biennale devança de quelques jours l'inauguration de la biennale officielle de Venise ${ }^{10}$. Des années plus tard, la galeriste organise un nouveau Micro-Salon pour soutenir une contre-manifestation de la Foire Internationale d'Art Contemporain, mais cette fois-ci 
dans une situation encore plus étonnante. En qualité de membre officiel du Comité d'Organisation pour la Foire Internationale d'Art Contemporain (CoFiac), et ce depuis la création de cette instance en $1975^{11}$, Iris Clert expose pour l'édition de 1980 en association avec la Galerie Marbach de Berne au stand n'M1 PATAK - Papiers nattés de Guy Houdouin (FIAC 1980 214-15). Cela ne l'empêche pas de participer parallèlement au Marché International des Galeries d'Art Moderne et Édition, pour lequel elle improvise au stand $n^{\circ} \mathrm{A} 12$ un Micro-Salon composé de cinquante exposants ${ }^{12}$ (Figure 2). Organisé au Pavillon d'exposition Bastille, ce Marché International des Galeries d'Art Moderne et Édition fonctionnait comme un Salon des Refusés de la FIAC. Ainsi, Iris Clert avait un pied dans la manifestation officielle et un autre dans un salon alternatif et contestataire.

Figure 2. Micro-Salon - 50 exposants, MIGAME. Marché International des Galeries d'Art Moderne et Éditions. Pavillon Bastille, Paris, Stand $n^{\circ} \mathrm{A} 12$ (19- 27 avril 1980). Catalogue d'exposition. Paris : SMI (1980), pages 96-97.

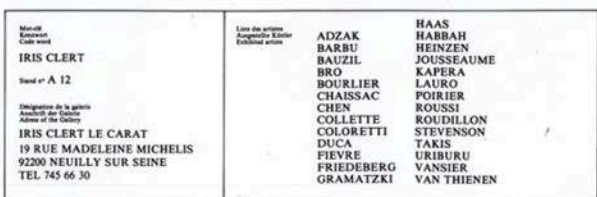

MICRO-SALON

50 EXPOSANTS

\section{IRIS CLERT}

Vous invite à visiter sa Nouvelle Galerie à Neuilly le CARAT* (Association loi 1901) 92200 Neuilly 7456630

\section{Déranger et dégenrer la société et l'art}

Le caractère ambigu de la position d'Iris Clert vis-à-vis du système de l'art l'expose parfois au mépris ou à la marginalisation, et, d'autres fois, lui permet d'accéder à la reconnaissance. La galeriste a pu déranger lorsqu'elle s'est amusée à révéler les ambivalences des mécanismes du monde de l'art et de la société. En ce sens, elle s'est toujours efforcée de mettre au défi la société dominante et ses normes, comme quand, évoluant dans une France patriarcale et conservatrice, elle prit la décision de divorcer pour vivre seule, et, comble de déraison pour une femme à l'époque, d'exercer un travail à son propre compte. C'est dans le même souci de préserver son indépendance qu'à l'heure des mouvements de revendications féministes du début des années soixante-dix elle inaugura en se disant « misogyne » (Clert 1974 n.p.) son Micro-Salon de 1974, sous-titré : Grandes Femmes petits formats ${ }^{13}$ (Figure 3). Organisée du 25 juin au 20 septembre 1974, cette manifestation s'inscrit dans la continuité de la programmation historique de la galerie en adoptant le format du Micro-Salon (Bergeret 2018). Il existe néanmoins une différence, et non des moindres, puisqu'elle est délibérément consacrée exclusivement à des œuvres réalisées par des femmes, de générations et d'orientations stylistiques différentes ${ }^{14}$. 
Figure 3. Catalogue d'exposition Grandes Femmes petits formats, Grandi donne piccolo formato, Great dames in small size, Grosse Künstlerinnen kleine werke. Micro-salon 1974, Paris, Galerie de l'Objet : Iris clert et Christofle, 12 rue Royale ( 25 juin -20 septembre 1974).

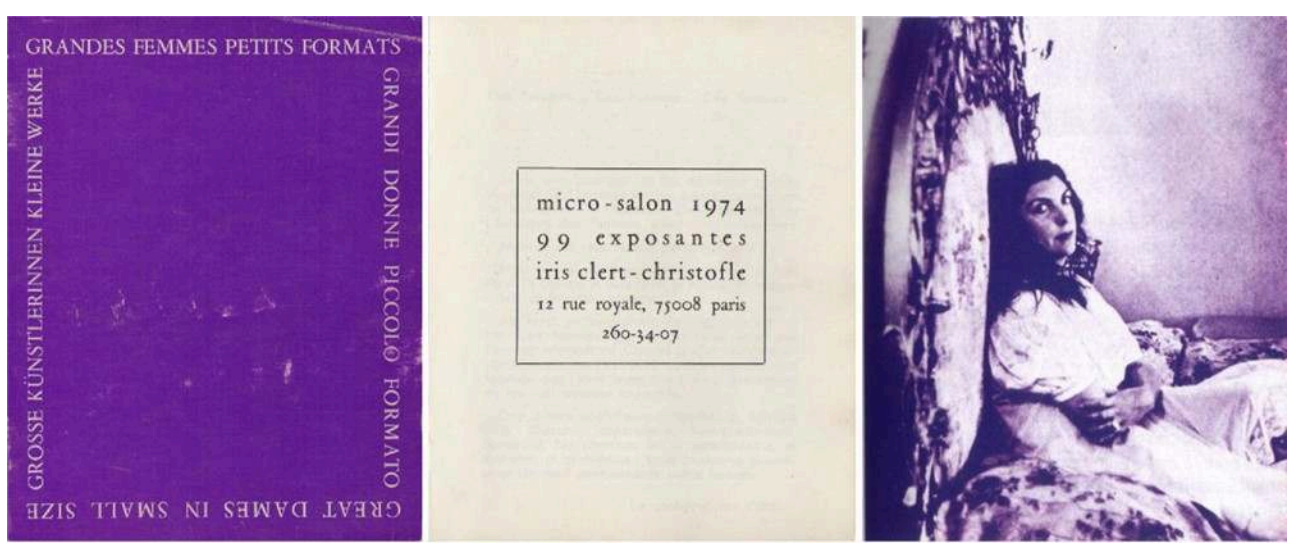

(c) Bibliothèque Kandinsky, MNAM/CCl, Centre Pompidou, Fonds Iris Clert.

Dans une partie de son livre Les femmes dans l'art, intitulée "Le retard de la France ", Marie-Jo Bonnet cite Grandes Femmes Petits Formats parmi les manifestations artistiques qui ont rendu compte de «la prise de conscience de la discrimination et de la nonreconnaissance des plasticiennes » (Bonnet 86) dans le contexte des avant-gardes en France. En effet, offrant un panorama de la production des artistes femmes du $\mathrm{XX}^{\mathrm{e}}$ siècle, des pionnières à la jeune génération, de Sonia Delaunay à Tania Mouraud, cette exposition apparaît rétrospectivement comme un acte fort, et demeure "un geste précurseur » (Gonnard et Lebovici 266) au sein de l'histoire de l'art. Une histoire de l'art qui la reconnait aujourd'hui comme une exposition pionnière à caractère féministe, organisée en France par une directrice de galerie d'art contemporain.

11 Il s'avère qu'après l'inauguration de ce Micro-Salon 1974, la presse place sa réception critique dans la perspective d'une lecture liée à une histoire féministe de l'art. Aline Dallier-Popper, écrit pour la revue Opus International un texte au début duquel elle remarque qu'il n'y avait pas eu d'exposition réunissant uniquement des artistes femmes à Paris depuis $1937^{15}$. Elle conclut en affirmant : "Quoi qu'il en soit, cette exposition vient enfin montrer que les artistes femmes existent aussi, et parfois manifestement " (Dallier-Popper 116). Le second article déterminant, parut dans Art in America, est signé Linda Nochlin (Nochlin 90). Au début de son article, la célèbre historienne de l'art américaine interroge le titre de l'exposition en se demandant s'il peut y avoir un lien intrinsèque entre petits formats et «feminine style ». Elle considère que cette association a pu être préjudiciable mais interprète finalement le petit format comme une expérimentation contemporaine défiant l'idée commune que le véritable art imposerait des productions de grandes dimensions. Elle place ainsi les œuvres de ces artistes femmes dans des préoccupations actuelles de l'art contemporain. L'indéniable dimension féministe de cette exposition n'apparait pourtant pas dans le texte de présentation de l'exposition rédigé par Iris Clert qui atteste des contradictions de ce parti pris :

Je ne sais pourquoi, le fait de réunir quatre-vingt-dix-neuf femmes semble à certains un geste de libération. De libération. De Mouvement de Libération des Femmes, avec trois majuscules. Mais je ne veux libérer personne, moi ! Mais je n'ai pas besoin d'être libérée. Je suis libre. Et comme je suis libre, je refuse les majuscules et les mouvements. Au fond, je suis plutôt misogyne, c'est un fait. Les 
femmes qui se prennent pour des Femmes m'embêtent. Comme je n'ai pas de principes, et comme j'ai trouvé quatre-vingt-dix-neuf femmes que j'aime bien, il m'a paru intéressant de les voir exposées ensemble. Ces quatre-vingt-dix-neuf femmes-là existaient déjà, chacune, séparément, individuellement, librement. $\mathrm{Ne}$ cherchez ici ni paternalisme, ni sexisme, ni problèmes : vous trouverez quatrevingt-dix-neuf passionnants petits formats. La centième, Iris Clert. (Clert 1974)

L'historienne de l'art Fabienne Dumont reste perplexe à la lecture de ces lignes, puisque si dans les faits l'exposition s'inscrit significativement dans une histoire de l'art féministe, elle s'en éloigne paradoxalement dans son discours, qui préfère le registre de l'ironie et «se camoufler sous couvert de misogynie " (Dumont 2014, 49). Iris Clert clame que, non sans trahir ses convictions, elle œuvre en faveur des femmes, à travers les femmes en tant qu'individus, en prenant toutefois le soin d'éviter toute sorte d'assimilation de son exposition à un groupe, et plus particulièrement au M.L.F., auquel elle fait explicitement référence. De telles considérations dérangent et ne correspondent pas à celles attendues de la part d'une femme qui participe malgré elle au moment historique que représentent les années soixante-dix et « où le champ artistique croise le mouvement de libération des femmes et s'en inspire » (Zabunyan 171). Grandes Femmes Petits Formats se révèle être, dans son acte, un modeste jalon de l'histoire d'un féminisme artistique en France, tout en reflétant à travers son discours les postures paradoxales d'Iris Clert (Bergeret 2018, 138).

\section{Micro-Salon International - II Micro-Salon di Iris Clert alla Galleria Apollinaire - Mini-School of Paris...}

13 S'il apparait comme une formule d'exposition dont Iris Clert use pour imposer des revendications anticonformistes, le concept de Micro-Salon prend également d'autres visages, tout aussi représentatifs des méthodes et pratiques élaborées par cette galerie d'avant-garde. Facilement transportable dans une valise, le Micro-Salon voyage dès l'année 1957 en Italie ${ }^{16}$ (Figure 4), puis les deux années suivantes à Bruxelles et à Berlin $^{17}$. 
Figure 4. Carton d'invitation à l'exposition collective Micro-Salon à la galerie Apollinaire. Milan (juin 1957).

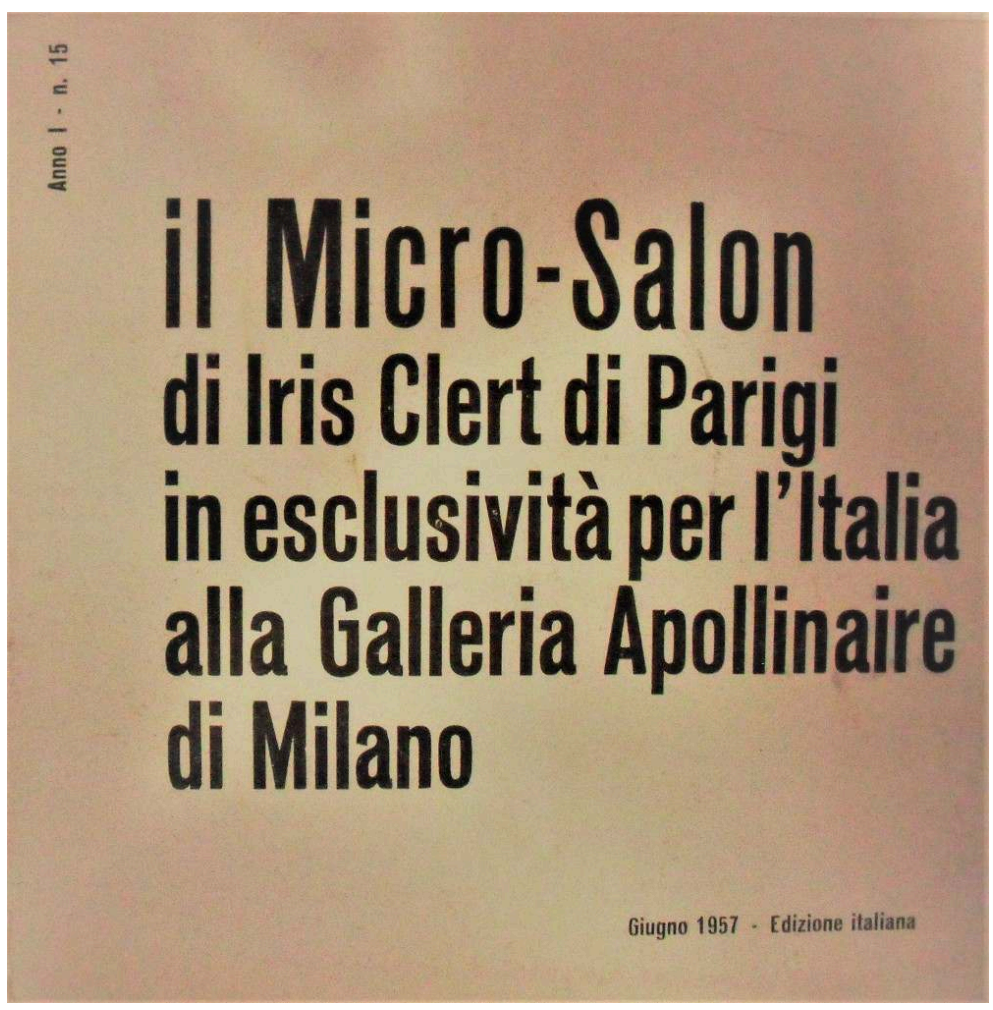

(c) Bibliothèque Kandinsky, MNAM/CCl, Centre Pompidou, Fonds Iris Clert.

Le Micro-Salon est la première exposition qui passe les frontières et contribue à l'internationalisation de la petite galerie parisienne. Alors que cette ambition se retrouve dans les premières intentions d'Iris Clert - «Mais je suis restée grecque, donc universelle. C'est pour cette raison que j'ai réuni autour de moi des aventuriers de l'art venus de tous les points du monde " (Clert 2003, 359) - cette volonté manifeste d'asseoir durablement le rayonnement international de sa galerie s'incarne aussi dans la multiplication des collaborations avec des galeries étrangères pour proposer les œuvres de ses artistes aux États-Unis (dans les galeries Richard Feigen et Dick Waddell de New York et la Gallery of Modern Art de Muriel Bigar à Scottsdale en Arizona) ${ }^{18}$, ou encore en Iran (avec la galerie Negar Afsaneh de Téhéran) ${ }^{19}$ et surtout en Belgique (avec les galeries Defacqz ${ }^{20}$, La Balance ${ }^{21}$ et Carrefour ${ }^{22}$ de Bruxelles et à Anvers avec la Galerie Swarte Panter $)^{23}$. Ce déploiement international s'affirme pleinement avec l'exposition anniversaire de la galerie qui adopte la forme d'un Micro-Salon intitulé Dix ans de passé, cent ans de futur, fêtée non pas à Paris mais à Stockholm, en la Galerie Eva af Burén, en février $1966^{24}$. Puis, c'est dans le cadre de la première Foire Internationale d'Art Contemporain (FIAC) en 1975 qu'Iris Clert réaffirme son ancrage dans le paysage international de l'art en proposant parmi les "one man show ", un Micro-Salon International, avec les «tableaux portables - paintings to wear " (FIAC 1975 109) de vingt $\operatorname{artistes}^{25}$ (Figures 5 et 6). 
Figure 5. Micro-Salon international - petits formats (la derrière nouveauté : le tableau portable paintings to wear). Paris, $2^{\mathrm{e}}$ Foire Internationale d'Art Contemporain, Pavillon d'expositions de la Bastille, Galerie Iris Clert : Stand $\mathrm{n}^{\circ} 108$ (30 janvier - 5 février 1975). FIAC 1975, cat. expo. (Paris, Pavillon d'expositions de la Bastille, 30 janvier - 5 février 1975), Paris : Organisation Idées Promotion (1975) pages 7 et 109.

\section{one man show}

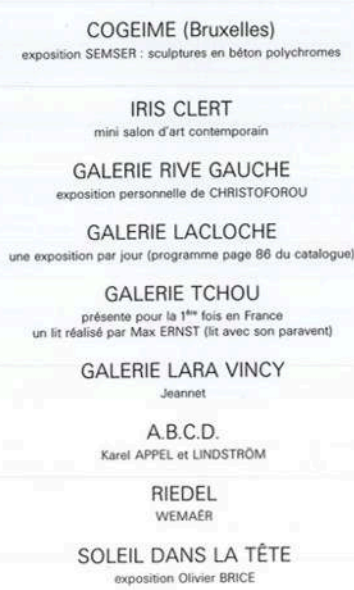

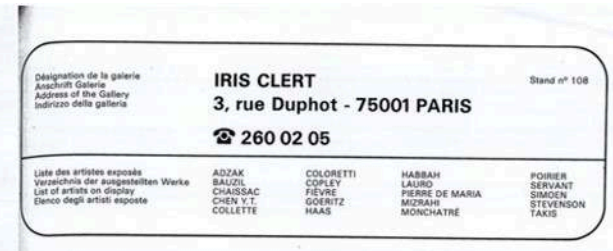

micro-salon international

petits formats

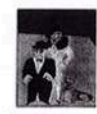

la demière nowveauté

le tableau portable paintings to wear

(c) Bibliothèque Kandinsky, MNAM/CCl, Centre Pompidou, Fonds Iris Clert.

Figure 6. Carton d'invitation à l'exposition collective Dix ans de passé, cent ans de futur. Stockholm, Galerie Eva af Burén (février 1966).

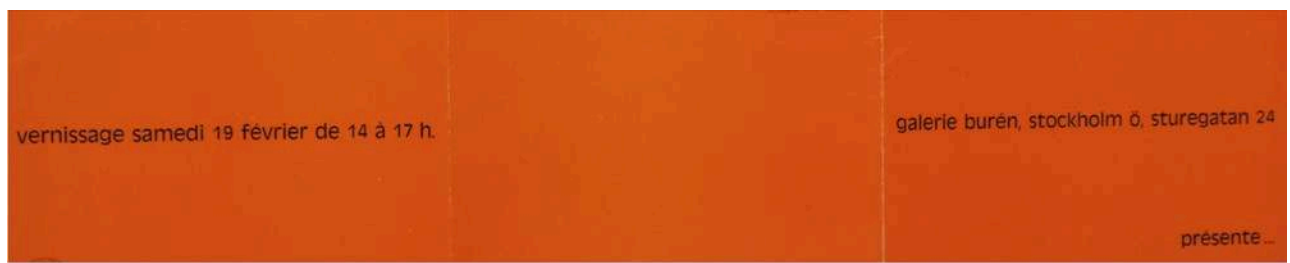

adzak arman bró bury chaissac copley dallegret fièvre fontana gelssler golub habbah hains klein kricke mack de maria manzoni piene quentin reinhardt stevenson soto takis tinguely tsingos vansier van hoevdonck van thienen adzak arman bró bury chaissac copley dallegret fièvre fontana geissler golub habbah hains klein kricke mack
de maria manzoni piene quentin reinhardt stevenson soto takis tinguely tsingos vansier van hoevdonck van thienen

(c) Bibliothèque Kandinsky, MNAM/CCl, Centre Pompidou, Fonds Iris Clert.

En renommant ainsi rétrospectivement les œuvres de petits formats qui composent ses Micro-Salons «tableaux portables », Iris Clert se souvient probablement de la grande liberté de mouvement et de la transportabilité que lui a offertes ce format d'exposition des années auparavant. S'il participe activement à la reconnaissance internationale de la galerie d'avant-garde, il ne connaît aucune frontière, comme dans le cas du MiniSchool of Paris, qui, initialement préparé pour être accroché à Dallas chez Nieman Marcus en 1966, a d'abord été présenté lors du voyage en mer sur le bateau United States Line qui conduit Iris Clert aux États-Unis (Figure 7). 
Figure 7. "Special edition for U.S.A., the most advanced art gallery in the world on the fastest liner on the seas, vernissage at sea October $1^{\text {st }} 12$ a.m. ». iris.time UNLIMITED 26 (Paris, Galerie Iris Clert, octobre 1966).

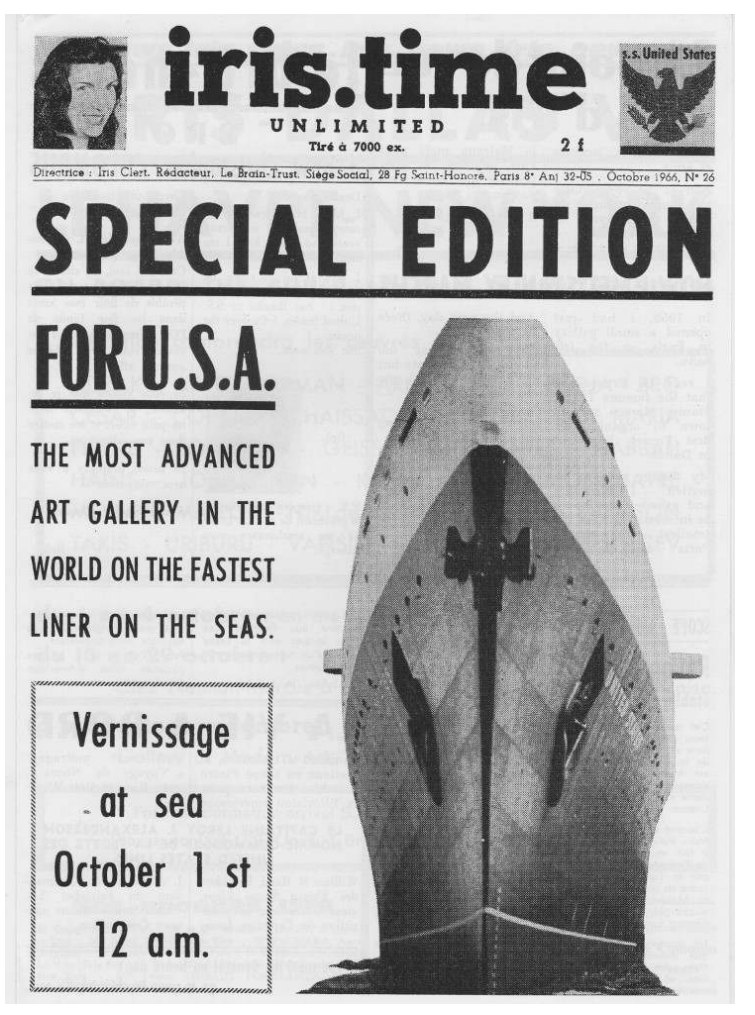

Lors d'un voyage précédent aux États-Unis, le Micro-Salon s'affranchit d'autres frontières, en parvenant à passer illégalement les douanes, sans les documents qui doivent normalement accompagner les œuvres d'art :

Dans sept valises, il y aura les restes du Micro-Salon, une cinquantaine de petits formats, mes robes, mes chaussures, mes manteaux et à tout hasard mes affaires d'été (bikinis compris) sans oublier mon fichier américain au grand complet. [...] A Idlewild Airport, Georges Staempfli vient m'accueillir. Il assiste d'un premier étage à l'arrivée des passagers aux prises avec les douaniers. Il me regarde de sa hauteur d'un air ironique, persuadé que j'aurai les pires ennuis avec mes multiples valises. Le douanier me demande :

- [...] ouvrez vos valises.

Je tremble. Il tombe justement sur celle qui est bourrée de petits tableaux.

- Qu'est-ce que c'est?

- Ce sont mes œuvres, je suis peintre.

- Vous comptez les vendre?

- Absolument pas. J'ai tellement d'amis aux Etats-Unis que j'ai apporté un cadeau pour chacun $[\ldots]$

- Ah ! bon. Alors passez. (Clert 2003, 207-8)

\section{Iris Clert Micro-Salonnière}

Quand en 1957 Iris Clert demande aux artistes de lui fournir des œuvres de petits formats pour composer son premier Micro-Salon, elle s'implique directement dans le processus de création de l'exposition qu'elle organise. C'est bien là l'une des caractéristiques remarquables de son action dans le monde des galeries d'art 
contemporain. Délaissant la dimension commerciale au profit de l'art, elle se fait créatrice de situations d'expositions inédites et originales tout en s'appropriant, d'une certaine façon, les œuvres des artistes qu'elle expose. Cette audace est celle à la fois d'une galeriste et d'une commissaire d'exposition (Glicenstein 2015), deux métiers au sein desquels des figures pionnières émergent ${ }^{26}$. En ce sens, certaines des méthodes et pratiques qu'elle élabore peuvent s'apparenter à celles des curateurs, tant la galeriste crée en son temps plusieurs expositions qui trouvent aujourd'hui des équivalents dans certaines propositions imaginées des années plus tard. Parmi elles, on retrouve encore l'un de ces Micro-Salons ${ }^{27}$. En novembre 1966, n'ayant pas vendu l'intégralité des œuvres de son Mini-School of Paris qu'elle avait présenté lors de la « Quinzaine française » chez Nieman Marcus à Dallas ${ }^{28}$, et à court d'argent pour rentrer à Paris, Iris Clert décide d'organiser en toute hâte une exposition «sauvage » dans sa suite à l'hôtel Chelsea de New York $^{29}$. Elle convoque tous ses collectionneurs américains à venir découvrir son mini-school of Paris à l'hôtel (Clert 2003, 344). La trivialité de cet événement saugrenu démontre les capacités d'anticipation de la galeriste. En effet, des années plus tard, dans un contexte bien différent, le commissaire d'exposition Hans-Ulrich Obrist organise, en 1993, une exposition de groupe dans la Chambre 763 de l'hôtel Carlton Palace à Paris ${ }^{30}$.

À l'image de quasiment toutes les expositions qu'Iris Clert présente, le format de MicroSalon compose un espace de création en constante réinvention. Véritable work in progress en son genre, il est une source d'émulation artistique permanente. Il revêt, entre 1957 et 1980, la forme d'une collection d'œuvres de petits formats en perpétuel renouvellement, puisque d'un Micro-Salon à un autre, des œuvres sont vendues et de nouvelles viennent l'enrichir. C'est également à travers les supports de promotion et de médiatisation de ces Micro-Salons, que se traduit la grande inventivité d'Iris Clert. Ainsi, l'une des particularités et originalités des affiches et cartons d'invitation conçus pour les Micro-Salons réside dans la forme et la composition inspirées par le format de l'exposition et les œuvres exposées. L'intention de réunir plus d'une centaine d'artistes pour le Micro-Salon d'Avril de 1957 apparait sur l'invitation (Figure 1) mais se retrouve également sur le carton d'invitation double face du Micro-Salon de 1958 ${ }^{31}$ (Figure 8) "plié en trois, qui, par ses dimensions réduites, et la liste interminable d'artistes exposées (elle se poursuit sur le verso) rend compte avec une formidable acuité des contraintes spatiales de l'exposition " (Airaud et V. Clert 19). Cette composition a été réutilisée en février 1966 pour le carton d'invitation de l'exposition collective du MicroSalon Dix ans de passé, cent ans de futur organisée à la galerie Eva af Burén de Stockholm ${ }^{32}$ (Figure 6). 
Figure 8. Carton d'invitation à l'exposition collective Micro-Salon 58. Paris, Galerie Iris Clert, 3 rue des Beaux-Arts (juillet - septembre 1958).

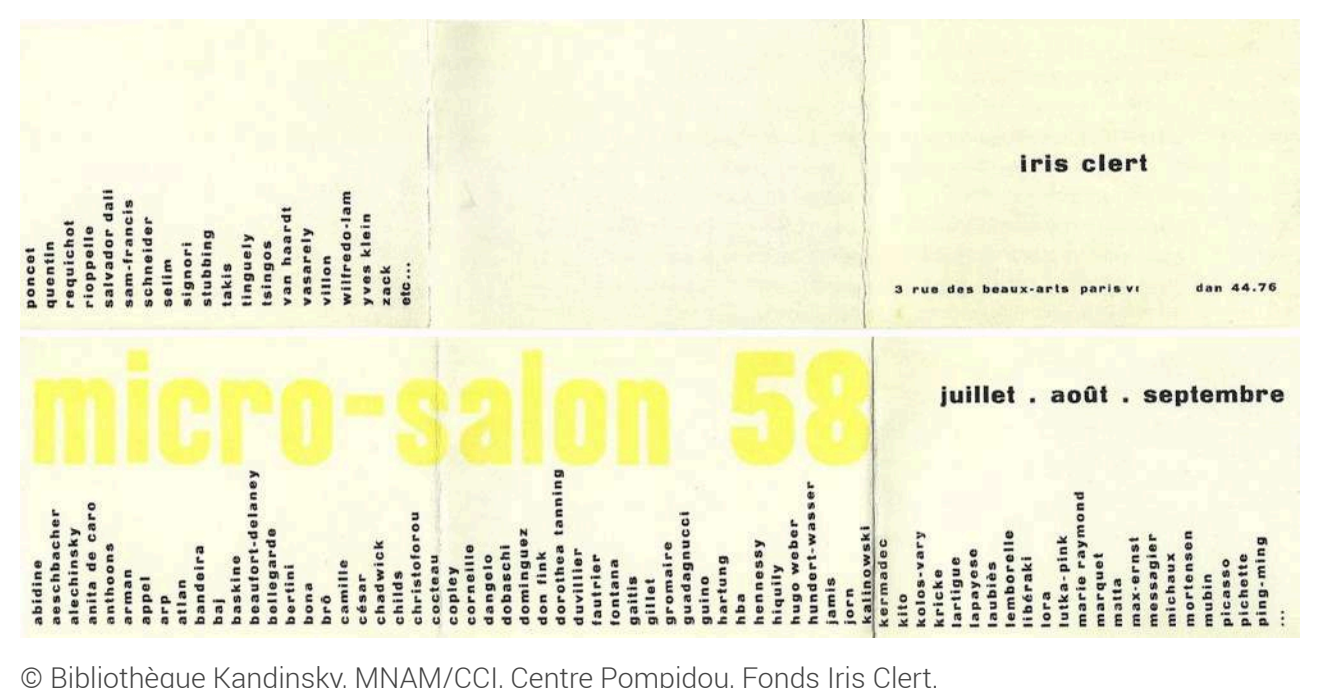

L'implication créatrice d'Iris Clert dans la communication va la pousser à s'octroyer le droit de créer et de changer l'identité de certains de ses artistes. Pour l'anecdote, c'est encore en lien avec les premiers Micro-Salons qu'Armand Fernandez, en avril 1958, devient « Arman ». La première fois que l'artiste est exposé par Iris Clert, c'est en 1957 lors du Micro-Salon d'avril : sur l'affiche-invitation son nom est orthographié Armand. L'année suivante, ce même Armand Fernandez n'est plus désigné de la même manière : une faute typographique de l'imprimeur aurait transformé son prénom en « Arman » et le nom a été supprimé33. Trouvant cette graphie originale et évoquant une identité d'Israélien (Clert 1975), Iris Clert décide de la réutiliser pour la première exposition personnelle de l'artiste en mai 1958 :

Qu'on change l'orthographe de mon nom me paraissait intolérable. J'ai fait un scandale. Je craignais qu'on ne me reconnaisse plus, j'avais peur d'avoir à reconstruire ma carrière. Iris Clert m'a alors pris à part : «Écoute, mon coco, tu es complètement inconnu. Tu as dix copains qui se souviennent de ton prénom. De plus, Armand cela fait garçon coiffeur. La prochaine exposition, on la fait aussi sous le nom d'Arman, ou on ne la fait pas. » C'est comme cela que je suis devenu Arman : [...] à cause d'Iris Clert. (Arman 191)

Devenu indissociable de la réception critique et historique de la galerie Iris Clert, le format d'exposition Micro-Salon prêta son nom à la modeste rétrospective qui fut consacrée à la galeriste au musée d'art contemporain de Strasbourg en 2003 : Iris Clert

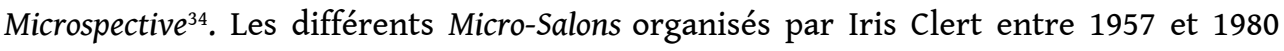
redéfinissent les fonctions des galeries d'art contemporain, notamment dans une demande de transformation du rapport à la marchandisation de l'art (Verlaine 452-56), et inscrivent son action singulière dans l'histoire de l'art (Bergeret 2017). À bien le considérer, ce format d'exposition compose un espace de revendication et de création alternatif en perpétuel réinvention, les petits formats des œuvres permettant une grande maniabilité et une liberté nouvelle.

21 Avant même de passer à la postérité et de s'inscrire dans une histoire des expositions, ce concept aura valu à Iris Clert des sollicitations, comme celle de la galerie allemande Hennemann qui lui écrit pour demander en 1977 la permission de copier ce format :

Vous avez organisé chez vous une exposition de petits formats sous le titre « microsalon »- devenue célèbre. Si je me souviens bien, c'était la première parution de ce 
mot ou de ce titre. Peut-être vous n'avez ni inventé ni trouvé ce « micro-salon »-il reste quand même tout à fait «IRISCLERTIEN». Je demande l'autorisation de réutiliser ce titre pour mon exposition de petits formats pour une exposition sur la vie artistique allemande de nos jours. (Fonds Iris Clert)

\section{BIBLIOGRAPHIE}

\section{Ouvrages cités}

AGOSTINELLI, Cristina. « Galerie Iris Clert ». 20 galeries du 20 siècle, France 1905-1970. Hors-série 2020 : Les Cahiers du Musée National d'art Moderne. Paris : Centre Pompidou, 2020. 92-95.

AIRAUD, Stéphanie et Vassili CLERT. Iris Clert Microspective. Cat. expo. (Strasbourg, Musée d'art contemporain, 7 novembre 2003 - 1 février 2004). Strasbourg : Musées de Strasbourg, 2003.

ARMAN. Arman galerie nationale du jeu de Paume. Cat. expo. (Paris, Galerie Nationale du jeu de Paume, 27 janvier au 12 avril 1998). Paris : RMN, 1998.

BERGERET, Servin. « 'Grandes Femmes petits formats - Micro-Salon 1974'. Une exposition de 99 artistes femmes organisée par la galeriste Iris Clert ». Communication dans le cadre de la journée d'étude « WAS - WOMEN ARTISTS SHOWS SALONS SOCIETIES : expositions collectives d'artistes femmes durant les années 1970 en Europe et Amérique du Nord » organisée par le laboratoire de recherche Artl@s (École normale supérieure, PSL, labex Transfers) et par l'association AWARE, avec la collaboration de la Terra Foundation for American Art. 14 juin 2018. URL : https:// vimeo.com/282807018 (page consultée le 25 mai 2021).

BERGERET, Servin. Iris Clert (1918-1986). Une galeriste singulière. Thèse de doctorat sous la direction de Valérie Dupont et de Bertrand Tillier. Dijon : Université de Bourgogne, 2017.

BERGERET, Servin. «Iris Clert: The territory and networks of a female gallery owner. Drawn from the exhibition Homage à Iris Clert, Grand Palais, Paris. Organised by Christiane de Casteras, June 1988 ». Women in Art and Literature Networks. Dir. Marianne Camus et Valérie Dupont. Cambridge Scholars Publishing : Newcastle-upon-Tyne, 2018. 130-142.

BONNET, Marie-Jo. Les femmes dans l'art. Paris : La Martinière, 2004.

CHUPIN-MORELLET, Dominique. Les petits formats de Maître Rey au Musée Rigaud. Perpignan : Ville de Perpignan ; Association des Amis du Musée Rigaud, 1995.

CLERT, Iris. Grandes Femmes petits formats - micro-salon 1974. Cat. expo. (Paris, Galerie de l'objet, 25 juin - 20 septembre 1974). Paris : Galerie Iris Clert et Christofle, 1974.

CLERT, Iris. iris time and life, mémoires sonores d'Iris Clert (six cassettes audios). Paris : Galerie Iris Clert, 1975.

CLERT, Iris. iris.time (l'Artventure). 1978. Paris : Denoël, 2003.

COMPAIN, Frédérique et Vassili CLERT. Iris-time le portrait d'une galeriste. Paris : Le Singe/Centre Georges-Pompidou/France 5, 2003. 
DALLIER-POPPER, Aline. « Grandes femmes et petits formats ». Opus International 53 (novembredécembre 1974) : 116 .

DAVALLON, Jean. L'exposition à l'œuvre. Stratégie de communication et de médiation symbolique. Paris : L'Harmattan, 2000.

D’ORGEVAL, Domitille. Le salon des Réalités Nouvelles. Les années décisives : de ses origines (1939) à son avènement (1946-1948). Thèse d'histoire de l'art contemporain sous la direction de Serge Lemoine. Paris : Université Paris IV - Sorbonne, 2007.

DUMONT, Fabienne. «À l'assaut ! Explosion d'expositions de femmes artistes en France pendant le mouvement féministe ». WAS (Women Artists Shows Salons Societies) : expositions collectives d'artistes femmes 1876-1976. Dir. Catherine Dossin, Camille Morineau et Hanna Alkema. Artl@s Bulletin 8:1 (2019). URL : https://docs.lib.purdue.edu/artlas/vol8/iss1/ (page consultée le 25 mai 2021).

DUMONT, Fabienne. Des sorcières comme les autres. Artistes et féministes dans la France des années 1970. Rennes : Presses Universitaires de Rennes, 2014.

Éloge du petit format. Peinture, sculpture, dessin. Collection Pierre Bourut. Cat. expo. (Saint-Germainen-Laye, Musée Municipal, 4 décembre 1978 - 29 janvier 1979). Saint-Germain-en-Laye : Musée Municipal de Saint-Germain-en-Laye, 1978.

FIAC 1975. Cat. expo. (Paris, Pavillon d'expositions de la Bastille, 30 janvier - 5 février 1975). Paris : Organisation Idées Promotion, 1975.

FIAC 1980. Cat. expo. (Paris, Grand Palais, 23 - 29 octobre 1980). Paris : Organisation Idées Promotion, 1980.

Fonds Iris Clert. Bibliothèque Kandinsky, Centre de documentation et de recherche du MNAM/ Cci, Centre Georges Pompidou, Paris.

GLICENSTEIN, Jérôme. L'art. Une histoire d'exposition. Paris : Presses Universitaires de France, 2009.

GLICENSTEIN, Jérôme. L'Invention du curateur. Mutations dans l'art contemporain. Paris : Presses Universitaires de France, 2015.

GONNARD, Catherine et Elisabeth LEBOVICI. Femmes artistes/artistes femmes - Paris, de 1880 à nos jours. Paris : Hazan, 2007.

Grandes Femmes petits formats - micro-salon 1974. Cat. expo. (Paris, Galerie de l'Objet, 25 juin - 20 septembre 1974). Paris : Galerie Iris Clert et Christofle, 1974.

MAINGON, Claire. Le Salon et ses artistes. Histoire des expositions du Roi Soleil aux Artistes français. Paris : Hermann, 2009.

MIGAME. Marché International des Galeries d'Art Moderne et Édition. Cat. expo. (Paris, Pavillon d'expositions Bastille, 19 - 27 avril 1980). Paris : SMI, 1980.

NOCHLIN, Linda. « Grandes Femmes Petits Formats at Iris Clert ». Art in America (JanuaryFebruary 1975) : 90 .

OBRIST, Hans-Ulrich. Hôtel Carlton Palace. Chambre 763. Cat. expo. (Paris, Hôtel Carlton Palace Chambre 763, 22 août - 25 septembre 1993). Stuttgart : Oktagon Verlag, 1993.

RAGON, Michel. 50 ans d'art vivant. Chronique vécue de la peinture et de la sculpture, 1950-2000. Paris : Fayard, 2001. 
ROIG SOTOMAYOR, Maria Antonia. Le Salon de Mai, un salon d'après-guerre. Fonctions et évolution de 1945 à 1970. Thèse de doctorat d'histoire de l'art sous la direction de François Robichon. Université Charles de Gaules - Lille 3, 2010.

VERLAINE, Julie. Les galeries d'art contemporain à Paris. Une histoire culturelle du marché de l'art, 1944-1970. Paris : Publications de La Sorbonne, 2012.

Yves Klein la révolution bleue. Paris, Film RMN, MK2TV et Centre Georges Pompidou, en association avec France 5, 2007.

ZABUNYAN, Elvan. « Histoire de l'art contemporain et théories féministes : le tournant de 1970 ». Genre, féminisme et valeur de l'art. Dir. Séverine Sofio, Perin Emel Yavuz et Pascale Molinier. Paris : L'Harmattan, 2007. 171-175.

\section{NOTES}

1. Propositions Monochromes, Yves Klein, Paris, Galerie Iris Clert, 3 rue des Beaux-Arts (10 mai - 25 mai 1957).

2. À l'image de Pierre Bourut, amateur invétéré de petits formats, dont la collection est dévoilée au Musée municipal de la ville de Saint-Germain-en-Laye en décembre 1978, sous le titre : Éloge du petit format. Peinture, sculpture, dessin. Collection Pierre Bourut.

3. Pour reprendre l'expression de Jean Davallon (Davallon 11).

4. Comme toute exposition ces Micro-Salons créent un discours sur l'art, dans le sens où « ce ne sont pas les œuvres d'art mais leur présentation qui fait émerger une pensée sur l'art - ou sur le monde - au sein d'une situation donnée » (Glicenstein 2009, 14).

5. Il faut néanmoins rappeler que les principaux Salons artistiques ont survécu pendant la guerre et demeurent des lieux d'échanges primordiaux, créant dans ces années difficiles un « lien intellectuel et social pour le monde des artistes » (Maingon 156).

6. Abstraction-Création est un collectif d'artistes fondé en 1931 par Auguste Herbin, Jean Hélion et Georges Vantongerloo.

7. 1955 : Éloge du petit format, galerie La Roue. Exposition organisée et préfacée par Michel Ragon (œuvres d'Atalan, Barré, Bergman, Bertrand, Camille, Corneille, Deyrolle, Doucet, Dumitresco, Fichet, Guitet, Hartung, Istrati, Koenig, Laubiès, Poliakoff, Schneider, Soulages, Sugaï, etc.) ; puis 1956 : Éloge du petit formats 2, galerie La Roue. Exposition organisée et préfacée par Michel Ragon (œuvres d'Atlan, Barré, Bergman, Bertrand, Bissière, Corneille, Dahmen, Deyrolle, Doucet, Dumitresco, Fautrier, Fichet, Guitet, Istrati, Koening, Maryan, Poliakoff, Reichel, Soulages, Wols) (Ragon 68 ; 73).

8. Micro-Salon d'Avril, Paris, Galerie Iris Clert, 3 rue des Beaux-Arts (12 avril - 8 mai 1957). CEuvres de Shafic Addoud, Abidine Dino, Yaacov Agam, Alcopley (Alfred Lewin Copley), Pierre Alechinsky, Anita De Caro, Avni Arbas, Armand Fernandez, Jean Arp, Benoist Baillargeau, Enrico Baj, Antonio Bandeira, Beauford Delaney, Claude Bellegarde, Frédéric Benrath, Etienne Beothy, Gianni Bertini, H. Bertrand, Boïlé, René Brô, Brown, Peter Brüning, Camille Bryen, Agustin Cardenas, César, Claude Chéreau, Bernard Childs, Guillaume Corneille, Harold B. Cousins, Jean Criton, Horia Damian, Jean Deyrolle, Jacques Delahaye, Dimitri, Jun Dobaschi, Hisao Domoto, Don Fink, Jacques Doucet, Max Ernst, Jean Fautrier, Jacques Favre de Thierrens, Marcelle Ferron, Lucio Fontana, Yannis Gaitis, Gauthier, Winfred Gaul, Gillet, Shirley Goldfarb, Pierre Graziani, Hella 
Guth, Richard Guino, Stanislas Halpern, Hans Hartung, Hba, Philippe Hossiasson, Fridensreich Hundertwasser, Eugène Ionesco, Egill Jacobsen, Jamis, Théophile Jaouen, Asger Jorn, Kam Zinchoom, Paul Klee, Fred Klein, Sigismond Kolos-Vary, Georges Koskas, Norbert Kricke, Antonio Lago Rivera, René Laubiès, Aglaé Libéraki, Liles, Lucatti, Frank Joseph Malina, Alfred Manessier, Charles Maussion, Marcel Polak, Jean Messagier, André Michaïlovitch Lanskoy, Richard Mortensen, Mozer, Orhon Mubin, Eugène Mollo, Kurt Neyers, Alicia Penalba, Pablo Picasso, Edouard Pignon, Lutka Pink, Prêcheur, Louis Pons, Bernard Quentin, Marie Raymond, Jean-Paul Riopelle, Ralph Rumney, Saint-Maur, Selim Turan, Gérard Schneider, Gustave Singier, Anna Staritsky, Tony Stubbing, Kumi Sugaï, Yasse Tabuchy, Takis Vassilakis, Jean Tinguely, Trufanov Mikhail Pavlovich, Nina Trygvadottyr, Tsingos Thanos, Dora Tuynman, Georges van Haardt, Boris Vansier, Victor Vasarely, Manuel Viola, François Willi Wendt, Wilfredo Lam, Yves Klein, Léon Zack et Zion.

9. Le jury ayant donné pour explication de ce rejet à Marie Raymond, la mère du peintre : «Vous comprenez, ce n'est pas vraiment suffisant tout de même ; alors si Yves acceptait au moins d'ajouter une petite ligne, ou un point, ou même simplement une tache d'une autre couleur, nous pourrions l'accrocher, mais une seule couleur unie, non, non, vraiment ce n'est pas assez, c'est impossible ! " (Yves Klein la révolution bleue).

10. Piccola Biennale, Palazzo Papadopoli, en marge de la Biennale de Venise (15 juin - 10 juillet 1962).

11. Cette association loi 1901 est fondée par Daniel Gervis, qui se charge de démarcher ses pairs pour participer et faire vivre ce nouvel outil. En plus de Daniel Lelong, élu président, et de la vice-présidente Denise René, Jean-Robert Arnaud est amené à officier en tant que secrétaire, et Pierre Nahon en tant que trésorier; les membres permanents comptent enfin Michel Durand-Dessert, Karl Flinker, Elisabeth Krief, Patrice Trigano et Iris Clert.

12. M.I.G.A.ME. : Marché International des Galeries d'Art Moderne et Edition, Paris, Bastille, Stand Iris Clert n¹2 (19 - 27 avril 1980). CEuvres de Roy Adzak, Louise Barbu, Gaby Bauzil, René Brô, Marc Bourlier, Gaston Chaissac, Chen Ying-Teh, Collette, Ferdinando Coloretti, Bernard Duca, Yolande Fièvre, Pedro Friedeberg, Eve Gramatzki, Raymond Haas, Abraham Habbah, Elga Heinzen, Dominique Jousseaume, Jean Kapéra, Georges Lauro, Jacques Poirier, Gilles Roussi, Jean Roudillon, Harold Stevenson, Takis Vassilakis, Nicolas Garcia Uriburu, Boris Vansier et Marcel van Thienen (MIGAME 97-98).

13. Grandes Femmes petits formats (Micro-salon 1974, Paris, Galerie de l'Objet : Iris clert et Christofle, 12 rue Royale (25 juin - 20 septembre 1974) : Eva Aeppli, Geneviève Asse, Thérèse Ampe-Jonas, Acerbi, Lee Burgess, Riva Boren, Janet Belden-Beyda, Esther Brunner, Gaby Bauzil, Louise Bentin, Bona Tibertelli de Pisis dite «Bona ", Pierrette Bloch, Martha Boto, Janice Biala, Anna Eva Bergman, Martine Boileau, Marcelle Cahn, Céline Chalem, Marta Colvin, Dumitriesco, Sonnia Delaunay, Diem Phung-Thi, Jos Decocq, Jeannie Dumesnil, Simona Ertan, Nadine Effront, Ruth Franken, Margarita Fanjul, Yolande Fièvre, Leonor Fini, Michèle Forgeois, Marianne Fayol, Shirley Goldfarb, Gill Goldsmith, Eve Gramatzki, Gabos, Ilse Getz, Polly Hope, Alice Hutchins, Elga Heinzen, Sara Holt, Hessie, Marianne Heske, Françoise Janicot, Shirley Jaffe, Ida Karskaya, Marina Karella, Lalan (Ching Lan van Thienen dite Lalan), Liliane Lijn, Warja Lavater, Nadia Leger, Ingeborg Luscher, Ivy Larsen, Claude Lalanne, Aglaé Libéraki, Caroline Lee, Agueda Lozano, Dominique Lorsch, Maria Manton, Margo, Joan Mitchell, Tania Mouraud, Cécile Muhlstein, Maglione, Annette Messager, Federica Marangoni, 
Jacqueline Monnier, Anne Mark, Sella Mertens, Joyce Mansour, Nicola, Aurélie Nemours, Louise Nevelson, Nikki de Saint-Phalle, Orvan, Orioli, Meret Oppenheim, Véga Pagava, Gina Pane, Lutka Pink, Nausica Pastra, Marta Pan, Marie Raymond, Bella Raftopoulo, Bridget Riley, Irène de Ramel, Vieira Da Silva, Marie-Josée Servant, Maria Simon, Gabriella Simossi, Dorothée Selz, Osa Sherdin, Seund Ja Rhee, Anne-Marie Soulcie, Day Schnabel, Léna Vandrey, Mireille Wunderli, Stella Waitzkin, Zuka et Louise Barbu.

14. En effet, se côtoient les noms d'artistes de veines figuratives comme Marie-Josée Servant, Eva Aeppli, Leonor Fini, et ceux de représentantes des langages abstraits purs, lyriques et autres : Anna-Eva Bergman, Shirley Goldfarb, Louise Nevelson, Aurélie Nemours. L'on croise par ailleurs les noms de performeuses telles que Françoise Janicot et Gina Pane, et pour finir plusieurs noms d'artistes expérimentant de diverses manières le textile comme médium ou sujet de représentations : Eve Gramatzki, Pierrette Bloch et Annette Messager.

15. Il faut nuancer cette affirmation d'Aline Dallier-Popper, qui semble oublier que l'Union des femmes peintres et sculpteurs a organisé des expositions de femmes artistes entre 1937 et 1974. À ce sujet, voir l'article de Fabienne Dumont qui donne le contexte féministe des expositions d'artistes femmes (Dumont 2019).

16. D’abord en juin à Milan (Galerie Apollinaire) puis en décembre à Rome (galerie la Tartagua).

17. Micro-Salon 58 (juillet - septembre 1958), Galerie Iris Clert. Puis, Micro-Salon Galerie Europe boulevard de waterloo-bruxelles (13 mars - 6 avril 1959), et enfin, Micro-Salon Kunstant Charlottenburg Platanenallee 16 (10 - 31 octobre 1959).

18. Peintures de Stevenson, Harold Stevenon, New York, Richard Feigen Gallery, en collaboration avec la Galerie Iris Clert (novembre 1963) ; 30 portraits du général de Gaulle, Boris Vansier, New York, Gallery Dick Waddell (novembre 1966) ; Peintures de René Brô, René Brô, Scottsdale, Gallery of Modern Art de Muriel Bigar (novembre 1966).

19. La Galerie Iris Clert expose les oeuvres de ses Artistes célèbres à la Galerie Negar Afsaneh, Roy Adzak, Arman, René Brô, Pierre de Maria, Yolande Fièvre, Abraham Habbah, Yves Klein, François Monchâtre, Jacques Poirier, Harold Stevenson, Nicolas Garcia Uriburu, Marcel van Thienen et Boris Vansier, Téhéran, Galerie Negar Afsaneh, 132 Avenue Soraya (octobre 1969) : "sur une invitation de Farah l'Impératrice d'Iran », pour reprendre les mots du carton d'invitation.

20. Deux expositions Galerie Defacqz d'Irène Hamerlinck à Bruxelles : Les Néoindividualistes (janvier 1965) puis Les Chromplexes de Jean-Claude Farhi, Baroques de Abraham Habbah, Le Cirque de Toto Meylan, et Les Jardins de Nicolas Garcia Uriburu (janvier 1968).

21. Peintures de René Brô, René Brô, Bruxelles, Galerie La Balance de Stéphane Janssen, en collaboration avec la Galerie Iris Clert (janvier 1968).

22. Les reliefs Platoniques, Roy Adzak, Les Boîtes, Yolande Fièvre, La Maison, Abraham Habbah et Les Palais de Banlieue, François Monchâtre, Bruxelles, Galerie Carrefour en collaboration avec la Galerie Iris Clert (janvier 1968).

23. Deux expositions de groupe Galerie de Swarte Panter à Anvers (décembre 1970 ; 28 septembre -8 octobre 1972).

24. Dix ans de passé, cent ans de futur, Stockholm, Galerie Eva af Burén (février 1966). Roy Adzak, René Brô, Pol Bury, Gaston Chaissac, Bill Copley, François Dallegret, Yolande 
Fièvre, Lucio Fotana, Klaus Geissler, Leon Golub, Abraham Habbah, Raymond Hains, Yves Klein, Norbert Kricke, Heinz Mack, Pierre de Maria, Piero Manzoni, Otto Piene, Bernard Quentin, Ad Reinhardt, Harold Stevenson, Jesús-Rafael Soto, Takis Vassilakis, Jean Tinguely, Tsingos Thanos, Boris Vansier, Paul van Hoeydonck, Marcel van Thienen. Stockholm, Galerie Eva af Burén.

25. "Micro-Salon international - petits formats (la derrière nouveauté : le tableau portable - paintongs to wear) ", $2^{e}$ Foire Internationale d'Art Contemporain, Stand $n^{\circ} 108$, Pavillon d'expositions de la Bastille, Paris (30 janvier - 5 février 1975). Roy Adzak, Gaby Bauzil, Gaston Chaissac, Chen Ying-Teh, Collette, Ferdinando Coloretti, Bill Copley, Yolande Fièvre, Mathias Goeritz, Raymond Haas, Abraham Habbah, Georges Lauro, Pierre de Maria, Jacques Mizrahi, François Monchâtre, Jacques Poirier, Marie-Josée Servant, Gérard Simoën, Harold Stevenson et Takis Vassilakis.

26. Les années d'activité de la Galerie Iris Clert sont celles de nombreux bouleversements rencontrés dans le monde de l'art contemporain, que ce soit au niveau de ses institutions, de ses modes de promotion et d'exposition. L'une des figures émergentes de ces redéfinitions contextuelles, est celle des commissaires d'exposition. Soutenus par d'importantes structures d'art contemporain indépendants, ces derniers vont favoriser la diffusion et la médiatisation de l'art de jeunes artistes. Parmi les précurseurs, l'histoire de l'art retient Harald Szeemann.

27. Pour donner quelques autres exemples pensons aux préparatifs de la Biennale Flottante de juin 1964, quand Iris Clert imagine une croisière en compagnie de collectionneurs, d'artistes et de leurs œuvres. Elle semble annoncer une situation expositionnelle se rapprochant de l'esthétique relationnelle, dont la conception peut évoquer celle d'une manifestation comme la vraie fausse biennale imaginée par Maurizio Cattelan en 2001 : 6th Caribbean Biennal, pour laquelle, sur le site de l'île antillaise de St. Kitts, il invita dix artistes dans un hôtel, afin de passer une semaine de vacances. En ce qui concerne la Biennale Flottante, l'idée de cette croisière prolongée par le choix d'exposer les œuvres dans un bateau sur le Grand Canal, pourrait faire penser aujourd'hui à la structure expositionnelle proposée en 2013 par l'artiste Joana Vasconcelos, qui, chargée de représenter le Portugal à la cinquante-cinquième Biennale de Venise, conçut un pavillon flottant : une navette fluviale lisboète entièrement recouverte d'azulejos.

28. Mini-School of Paris, "Quinzaine française » chez Nieman Marcus, Dallas (octobre 1966). Avec des œuvres de Roy Adzak, Karel Appel, Arman, François Arnal, Enrico Baj, Erik Borja, René Brô, César, Juan-Manuel Colina, Gaston Chaissac, François Dallegret, Pierre de Maria, Yolande Fièvre, Lucio Fontana, Klaus Geissler, Juan Genovés, Abraham Habbah, Raymond Hains, Erling Johansson, Yves Klein, John Levee, François Monchâtre, Marcel Meylan dit "Toto Meylan", Ronald Mallory, Niki de St-Phalle, Jesús-Rafael Soto, Harold Stevenson, Takis Vassilakis, Nicolas Garcia Uriburu, Boris Vansier, Victor Vasarely, Wols et Zev.

29. Mini-school of Paris, dans une suite de l'hôtel Chelsea, New York (novembre 1966(.

30. Hôtel Carlton Palace. Chambre 763, Paris, Hôtel Carlton Palace - Chambre 763 (22 août 25 septembre 1993).

31. Micro-Salon 58, Paris, Galerie Iris Clert, 3 rue des Beaux-Arts (juillet - septembre 1958). Dino Abidine, Arthur Aeschbacher, Pierre Alechinsky, Anita de Caro, Willy Anthoons, Arman, Karel Appel, Jean Arp, Jean-Michel Atlan, Antonio Bandeira, Enrico Baj, Maurice Baskine, Beauford Delaney, Claude Bellegarde, Gianni Bertini, Tibertelli de 
Pisis dite "Bona ", René Brô, Camille Bryen, César, Lynn Chadwick, Bernard Childs, John Christoforou, Jean Cocteau, Bill Copley, Guillaume Corneille, Sergio Dangelo, Jun Dobaschi, Oscar Dominguez, Don Fink, Dorothea Tanning, René Duvillier, Jean Fautrier, Jacques Favre de Thierrens, Lucio Fontana, Yannis Gaitis, Gillet, Marcel Gromaire, Gigi Guadagnucci, Richard Guino, Hans Hartung, Hba, Timothy Hennessy, Philippe Hiquily, Hugo Weber, Fridensreich Hundertwasser, Eugène Ionesco, Jamis, Asger Jorn, Horst Egon Kalinowski, Eugène-Nestor de Kermadec, Akira Kitô, Sigismond Kolos-Vary, Norbert Kricke, Guy Lartigue, José Ramón Lapayese, René Laubiès, Guy Lemborelle, Aglaé Libéraki, Silvano Lora, Lutka Pink, Marie Raymond, Albert Marquet, Roberto Matta, Max-Ernst, Jean Messagier, Henri Michaux, Richard Mortensen, Orhon Mubin, Pablo Picasso, James Pichette, Hsiung Ping-ming, Antoine Poncet, Bernard Quentin, Bernard Requichot, Jean-Paul Riopelle, Salvador Dali, Sam Francis, Gérard Schneider, Selim Turan, Ilio Signori, Tony Stubbing, Takis Vassilakis, Jean Tinguely, Tsingos Thanos, Georges van Haardt, Victor Vasarely, Jacques Villon, Wilfredo Lam, Yves Klein, Léon Zack, etc.

32. Dix ans de passé, cent ans de futur, Stockholm, Galerie Eva af Burén (février 1966).

33. Dans le film de Frédéric Campain et Vassili Clert, Arman rappelle bien qu'Iris Clert est à l'origine de son nom d'artiste et soutient qu'il s'agit au départ d'une erreur de l'imprimeur (Compain et V. Clert).

34. Iris Clert Microspective, expo. (Strasbourg, Musée d'art contemporain, 7 novembre $2003-1^{\text {er }}$ février 2004).

\section{RÉSUMÉS}

Cet article propose d'étudier les différents Micro-Salons organisés par la galerie d'art contemporain dirigée par Iris Clert entre 1957 et 1980, en s'attachant à restituer la réception critique de ces expositions en leur temps, tout en déterminant leur valeur historique. L'analyse montre que ces Micro-Salons composent un concept d'exposition qui offre une grande liberté reposant exclusivement sur le petit format des œuvres exposées, tout en révélant l'action de véritable commissaire d'exposition dont a fait preuve Iris Clert qui exploita le champ des possibles de ce concept aussi inédit qu'innovant.

This article examines the various "Micro-Salons" that were held in the contemporary art gallery managed by Iris Clert between 1957 and 1980. It explores the critical reception of these exhibitions in their time and seeks to determine their historical value. It discusses how these "Micro-Salons" constitute an exhibition concept that offers a great freedom based exclusively on the small format of the works exhibited, while revealing Iris Clert's practice as a proper curator who exploited the range of possibilities of a concept which was both original and innovative. 


\section{INDEX}

Keywords : Clert (Iris), contemporary art gallery, history of exhibitions, salon, small formats, international contemporary art fair, women in art, curating, avant-garde, micro-salon Mots-clés : Clert (Iris), galerie d'art contemporain, histoire des expositions, salon, petits formats, foires internationales d'art contemporain, femmes dans l'art, commissaire d'exposition, avant-garde, micro-salon

\section{AUTEUR}

\section{SERVIN BERGERET}

Laboratoire Interdisciplinaire de Recherche LIR3S « Sociétés, Sensibilités, Soin », UMR 7366 CNRS-Université de Bourgogne

Servin Bergeret est docteur en histoire de l'art contemporain et qualifié aux fonctions de maître de conférences (22e section CNU). Il enseigne l'histoire de l'art à la Haute École des Arts du Rhin à Mulhouse, à l'École des Beaux-Arts de Beaune et à l'Université de Bourgogne-Franche-Comté. Chercheur associé au sein du Laboratoire Interdisciplinaire de Recherche LIR3S « Sociétés, Sensibilités, Soin », UMR 7366 CNRS-uB, ses recherches portent sur les galeries d'art contemporain à Paris depuis le milieu du XIXe siècle, l'histoire des expositions, les femmes dans l'art et les mutations artistiques des années 1950 à nos jours. Il est l'auteur d'une thèse sur la galeriste Iris Clert mais également de plusieurs articles sur des aspects de la galerie Iris Clert, ainsi que sur des œuvres d'artistes dont Hervé Guibert, Andres Serrano, Joana Vasconcelos et Virginie Marnat. 